\title{
Robot-Assisted Hybrid Laparoscopic Roux-en-Y Gastric Bypass: Surgical Technique and Early Outcomes
}

\author{
Subhashini M. Ayloo, MD, FACS, Pietro Addeo, MD, Galaxy Shah, MD, \\ Fabio Sbrana, MD, FACS, and Pier Cristoforo Giulianotti, MD, FACS
}

\begin{abstract}
Background: Roux-en-Y gastric bypass performed laparoscopically remains the gold standard in bariatric surgery. The role of robot-assisted laparoscopic Roux-en-Y gastric bypass has not been clearly defined.

Methods: We present 80 consecutive cases of robot-assisted laparoscopic Roux-en-Y gastric bypass performed at a single institution. Mechanics, early outcomes, and learning curve are evaluated. Eighty robot-assisted laparoscopic Roux-en-Y gastric bypasses were performed on 71 women and 9 men with a mean age of 39 years, mean preoperative weight of $134 \mathrm{~kg}$, and mean BMI of 48 .

Results: Total mean operative time was 209 minutes. There was no mortality, leak, stricture, or obstruction. Conclusion: Robot-assisted laparoscopic Roux-en-Y gastric bypass is a safe and feasible option for bariatric surgery. Its role in improving surgical outcomes needs to be defined further.
\end{abstract}

\section{Introduction}

$\mathbf{R}$ OUX-EN-Y GASTRIC BYPASS is the most commonly performed procedure in the United States for the surgical treatment of morbid obesity. ${ }^{1}$ It was first performed openly in 1967 by Mason and Ito, ${ }^{2}$ and then reproduced laparoscopically by Wittgrove et al. in $1994 .^{3}$ The laparoscopic technique showed clear advantages over the open method and quickly gained momentum with a significant increase in cases from 13,386 in 1998 to 121,055 procedures performed in $2004 .^{4}$

Although laparoscopy is now the accepted approach to Roux-en- $Y$ gastric bypass, it requires advanced laparoscopic skills, including fluent suturing, intra-corporeal knot-tying, and the ability to work with linear instruments in multiple quadrants. The procedure has a steep learning curve with varying ranges of mortality. 5,6

With the introduction of robotic surgery in recent years, the number of applications in general surgery increased significantly. Its role in bariatric surgery, however, has not grown as significantly and still needs to be defined. ${ }^{7,8}$ The failure of robotic surgery to gain popularity in its application to gastrointestinal surgery is multifactorial: (1) cost, (2) challenge in objectively measuring the contribution of the robot for the benefit of patient, surgeon, and the procedure, (3) specialized team fluent with the robotic system, (4) tedious setup with docking and undocking for multiquadrant access, and (5) lack of studies establishing cost versus patient benefit. Preliminary experience published by Parini et al. has shown the mean operative time to be about 201 minutes (range, 90-300 minutes) with no anastomotic leak, fistula, bleeding, and mortality. The authors concluded with standardization of the robotic technique it is possible to reduce the operative time. ${ }^{9}$ This article describes a standardized surgical technique for robotassisted laparoscopic Roux-en-Y gastric bypass and presents the early outcomes in a series of 80 consecutive patients.

\section{Methods}

Between July 2007 and May 2009, 80 consecutive morbidly obese patients underwent robot-assisted laparoscopic Rouxen-Y gastric bypass by a single surgeon at the University of Illinois at Chicago. All patients were preoperatively evaluated by a multidisciplinary team including an internist, endocrinologist, nutritionist, psychologist, and bariatric surgeon. Patients underwent medically supervised weight loss and psychological clearance before their surgery. All patients were informed of the risks inherent with bariatric surgery, as well as the potential benefits, and a written consent was obtained by all.

There were a total of 9 men and 71 women with a mean age of 39 years (range 23-59 years). The mean preoperative weight was $134 \mathrm{~kg}$ (range 95-204 kg) and the mean BMI was $48 \mathrm{~kg} / \mathrm{m}^{2}$ (range $35-63 \mathrm{~kg} / \mathrm{m}^{2}$ ). Data were collected prospectively in a dedicated bariatric database. Clinical parameters included patient characteristics, operative variables, and

Division of General, Minimally Invasive, and Robotic Surgery, Department of Surgery, University of Illinois at Chicago, Chicago, Illinois. 
short-term outcomes. Patient characteristics included age, gender, preoperative BMI, and presence and type of comorbidities. Operative data included operative time, which was calculated as time between pneumoperitoneum induction and port-site closure. Early morbidity and mortality were defined as occurring 3 months after surgery.

Patients were positioned supine with both arms tucked and the operating table positioned slightly rotated $\sim 15^{\circ}$ on the left side. Pneumoperitoneum was achieved with a Veress needle in the left upper quadrant. The first 12-mm trocar (trocar 1) was placed under direct observation just to the right of the umbilicus (ENDOPATH ${ }^{\circledR}$ XCEL ${ }^{\mathrm{TM}}$ Bladeless trocar; Ethicon Endo-Surgery, Somerville, NJ). Two other 12-mm trocars were placed on the midclavicular line (trocar 2) and anterior axillary line (trocar 3) in the left upper quadrant. Another $12-\mathrm{mm}$ trocar was placed at the right midclavicular line (trocar 4), and one 5-mm trocar (trocar 5) was placed at the right upper quadrant, as shown in Figure 1. A Nathanson liver retractor was used to elevate the left lobe of the liver.

For the laparoscopic phase of the procedure, the right-sided trocars were used as the surgeon's access and the left sided trocars as the first-assistant's access to create a $30 \mathrm{~mL}$ gastric pouch. After trocar placement, the operating table was positioned in reverse Trendelenburg. The lesser sac was entered by creating a window in the gastrohepatic ligament. The gastric pouch was created using an Endostapler to staple and transect the stomach, starting $\sim 5 \mathrm{~cm}$ from the gastroesophageal junction on the lesser curvature. The transection of the stomach was performed horizontally and then vertically toward the Angle of His, using a 32-Fr bougie as a guide. The operating table was positioned level and the transverse mesocolon retracted cranially with identification of the ligament of Treitz. The jejunal loop was identified at about $50 \mathrm{~cm}$ distal to the ligament of Treitz, then stapled, and transected with an Endostapler. The Harmonic shears were used to transect its mesentery. Approximately $150 \mathrm{~cm}$ of the Roux limb was bypassed, and the afferent and efferent limbs were anastomosed side-to-side using a 45-mm white cartridge endostapler (Ethicon Endo-Surgery). The common enterotomy was

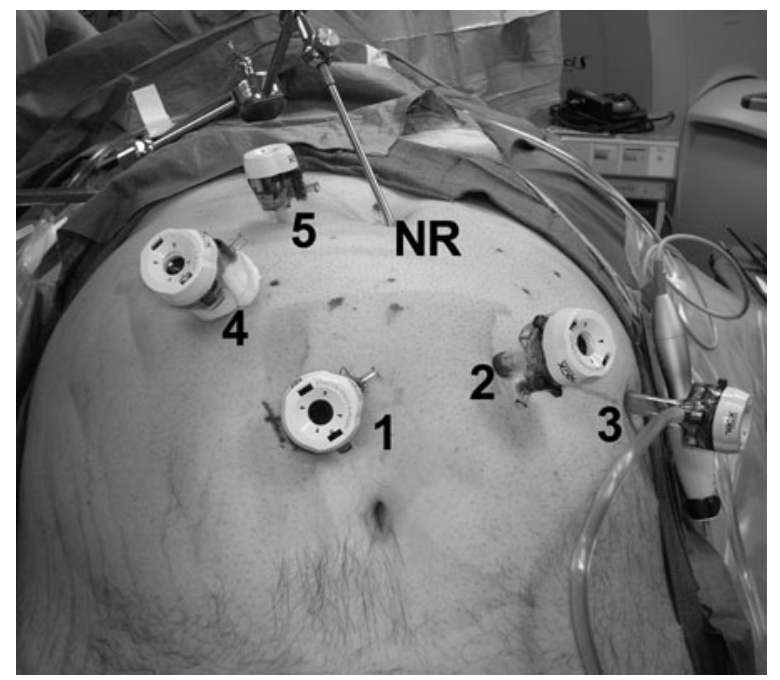

FIG. 1. Trocar positioning. NR, Nathanson liver retractor. handsewn in two layers, and the mesenteric defect closed using 2-0 silk suture.

To begin the robotic phase of the procedure, the operating table was positioned in slight reverse Trendelenburg and the da Vinci robotic surgical system (Intuitive, Sunnyvale, CA) cart docked cranially. A $30^{\circ}$ camera scope was inserted face down through trocar 2, and the robotic trocar was attached to the robotic arms that were inserted into trocars 3 and trocar 4 using a trocar-inside-trocar technique (Fig. 2). Using a grasper and needle driver, the Roux limb was robotically handsewn to the gastric pouch. The needle driver was removed and robotic harmonic shears were used to create an enterotomy and a gastrotomy. The robotic harmonic shears were replaced with the needle driver, and the anastomosis was handsewn in two layers using a 2/0 PDS suture $\sim 15 \mathrm{~cm}$ long. The robotic system was undocked, and an endoscopy performed to check the integrity of the gastrojejunal anastomosis.

In the last 10 procedures performed, the jejunojejunostomy was created robotically to move toward the goal of completing the entire technique robotically. This was done by positioning trocars 2 and 3 at the lower end of the left midabdomen. The gastric pouch was created as described previously, and the robotic cart was docked cranially. The proximal jejunum was grasped at about $70 \mathrm{~cm}$ from the ligament of Treitz and brought out to the gastric pouch via the antecolic antegastric route. A two-layered handsewn gastrojejunostomy anastomosis was performed using a 15- $\mathrm{cm} \mathrm{2/0}$ PDS suture (Ethicon Endo-Surgery).

After completion of the gastrojejunal anastomosis, the jejunum was traced $\sim 150 \mathrm{~cm}$ distally. The distal jejunal loop was brought out close to the proximal jejunum $(40-50 \mathrm{~cm}$ from the ligament of Treitz), and a stay suture was placed to align the bowel loops. An enterotomy was created in both

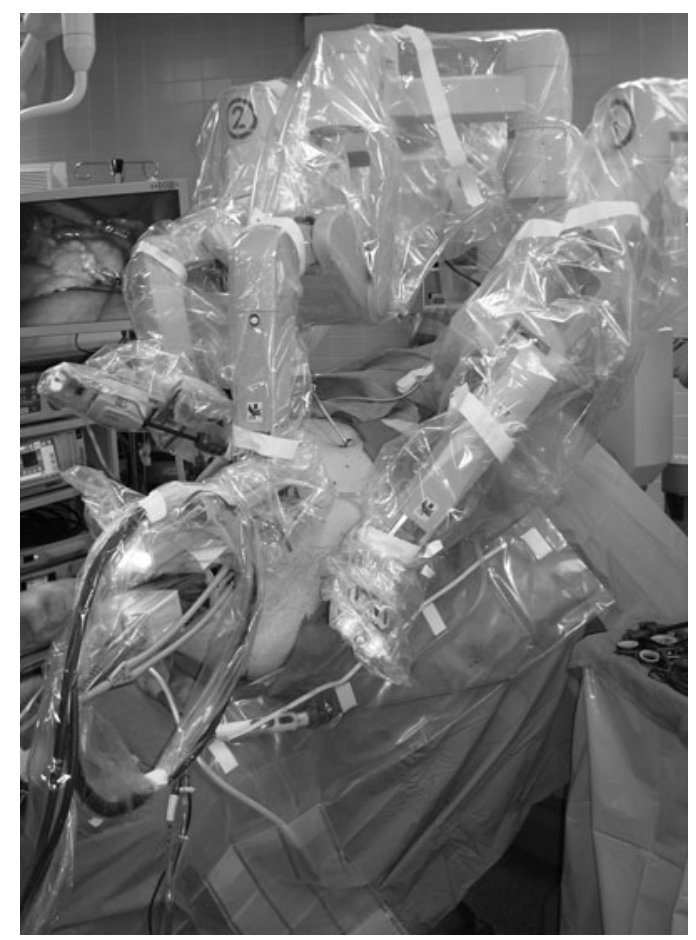

FIG. 2. Robotic setup. 
bowel loops using harmonic shears inserted via trocar 3 . The robotic arm was then detached from trocar 3 and an endostapler passed through to create a side-to-side jejuno-jejunal anastomosis. The common enterotomy was closed in a twolayer fashion using a 2/0 PDS suture. A mesenteric window was created close to the gastrojejunal anastomosis using a grasper for gentle blunt dissection. An endostapler was then brought in through trocar 1 to staple and transect the bridging bowel between the gastrojejunostomy and the jejunojejunostomy.

\section{Results}

The median operative time was 209 minutes (range 129-340 minutes). There was no mortality in this series. One patient required reoperation for pyriform sinus perforation from difficult intubation that was drained, and another patient required conversion to open surgery from stapler enterotomy inserted from patient side assistant. There were no reports of gastrointestinal leak, gastrojejunal stricture, gastrogastric fistula, or gastrointestinal obstruction. The average length of stay was 2 days (range 1-6 days).

\section{Discussion}

Roux-en-Y gastric bypass requires swift two-handed manipulation of tissues combined with an understanding of the orientation in multiple quadrants. It also requires fluent suturing abilities and intracorporeal knot tying skills needed to perform anastomosis. In traditional open surgery, these actions can typically be performed with ease; however, performing these maneuvers via conventional laparoscopy can be technically challenging. Most surgeons have learned this new set of skills, with varying levels of mastery. Still others have modified the technique with the assistance of stapling devices and staple line reinforcement material, along with suturing devices designed to reduce the amount of handsewn suturing required for a given procedure. ${ }^{10,11}$

Conventional laparoscopic technology brings with it limitations inherent in a two-dimensional visual system with linear instruments that provide no articulation and offer a varying degree of torque with which to work in multiple quadrants. As a result, the feasibility of obtaining precise reproducible movements is also limited, particularly in high BMI patients. This invariably leads to conversion to traditional open surgery, and on some occasions, complications arise postoperatively. ${ }^{12}$ Mortality rate ranged from $0.23 \%$ to $2 \%$, resulting from early complications of pulmonary embolism, anastomotic leak, and hemorrhage; furthermore, late complications of obstruction, anastomotic stenosis, fistula, ulcer, and incisional hernia have all been documented. ${ }^{13-15}$

The technical advantages offered by the da Vinci Surgical System make it a valid alternative to the laparoscopic approach for Roux-en-Y gastric bypass. We believe that the results presented in this series of 80 patients confirm its feasibility and safety. In fact, the operative technique for performing the robotic Roux-en- $Y$ gastric bypass is not significantly different from the conventional laparoscopic technique, as described by Higa et al., or robotically by Mohr et al. ${ }^{16,17}$ Several key technical points, however, should be emphasized to ensure that the procedure is performed effectively. First and foremost, we recommend beginning the procedure with the trocar-inside-trocar technique described above. By doing so, this employs the same port placement to which the surgeon became accustomed to previously and simply modify it to prevent external collision of the robotic arms. To do this, we have found that three things are essential: (1) a dedicated team that is experienced in the use of robotic technology; (2) correct positioning of the operating table for the robot to be docked swiftly; and (3) the robotic arms draped before the start of the procedure. This ensures that when it is time for the robotic portion of the operation, the only remaining thing to be done is to dock the robot to the operating table and attach the robotic arms with instruments using the previously placed trocars.

Other authors have demonstrated the importance of following these key steps as well. By doing so, the robotic setup time is significantly decreased, thereby addressing one of the major hindrances for surgeons when switching to the robotic system. ${ }^{17}$

The hybrid technique described here represents a preliminary step toward performing a fully robotic Roux-en-Y gastric bypass. Further, by standardizing this procedure, more surgeons would likely adopt the technique. Of course, the advantage to performing the entire procedure robotically is that it eliminates the need to move back and forth from laparoscopic to robotic in the middle of the procedure. It also allows for better ergonomics when performing both anastomoses robotically. The hybrid technique would still require advanced laparoscopic skills to perform the jejunojejunostomy and closure of the mesentery afterward. To perform the entire procedure robotically, trocar 3 would have to be placed very laterally on the left mid-abdomen or slightly to the left lower abdomen. Because the jejunojejunostomy is temporally located in the left mid-abdomen, positioning the trocar in this way provides additional room in the abdomen to bring in an endoscopic stapler to create the anastomosis. It is also important to keep the correct orientation of the bowel loops to avoid anastomosing the wrong loops. To avoid this, at least in the learning phase, it is crucial to tag the afferent and efferent limbs at the gastrojejunostomy and jejunojejunostomy anastomosis site.

Performing a totally robotic procedure gives the console surgeon more independence and requires minimal assistance from the first assistant. With this, an assistant with little laparoscopic skills can still assist in the procedure, but with less interruption. Additionally, the single docking technique allows for the least disruption in the fluency of the case.

There are reports documenting a learning curve of at least 100 cases for laparoscopic gastric bypass by an experienced surgeon. ${ }^{18,19}$ There are fewer reports from robotic studies, and those have indicated 10-15 cases as the learning curve for robotic gastric bypass. ${ }^{17}$

Reports also show that robotic Roux-en-Y gastric bypass can be safely performed with lower operating times and fewer complications than those obtained with laparoscopic gastric bypass. $^{20,21}$ On the contrary, Hubens et al. published their experience with the da Vinci robot system on 45 morbidly obese patients to perform Roux-en-y gastric bypass and noted total operative time to be shorter for laparoscopic group: mean robotic setup time of 30 minutes and more conversions to open surgery in the robotic group. In spite of this, there was no difference in postoperative complications in both robotic and laparoscopic groups in terms of leakage and stenosis. ${ }^{22}$ 
Our preliminary results indicate that the outcomes and complications are comparable to the laparoscopic approach. ${ }^{23}$ This may be in part due to the ease of using the system and its advantages over conventional laparoscopy, as well as its reproducibility. Recent reports have also addressed the issue of costs associated with the robotic system for different procedures. $^{24}$ This issue needs to be addressed side by side with the benefits to the patient and surgeon. Robotic technology can increase the threshold for conversion to open procedure, particularly in high BMI patients, and can potentially decrease morbidity related to gastrointestinal leak and gastrojejunal stricture, with a shortened learning curve. Additionally, there are benefits to the console surgeon in terms of improved ergonomics to perform the procedure.

Robot-assisted laparoscopic Roux-en-Y gastric bypass is a technical option that can easily be standardized and added to the field of bariatric surgery. The role of the robot has the potential to make the operation ergonomically easier, reproducible, precise, and efficient and to shorten the learning curve. Its role as it relates to potentially significant outcomes still needs to be defined.

\section{Disclosure Statement}

No competing financial interests exist.

\section{References}

1. Tice JA, Karliner L, Walsh J, et al. Gastric banding or bypass? A systematic review comparing the two most popular bariatric procedures. Am J Med 2008;121:885-893.

2. Mason EE, Ito C. Gastric bypass in obesity. Surg Clin North Am 1967;47:1345-1351.

3. Wittgrove AC, Clark GW, Tremblay LJ. Laparoscopic gastric bypass, Roux-en-Y: Preliminary report of five cases. Obes Surg 1994;4:353-357.

4. Zhao and Encinosa, Bariatric Surgery Utilization and Outcomes in 1998 and 2004, HCUP Statistical Brief \#23, January 2007. Agency for Healthcare Research Quality, Rockville, MD.

5. Podnos YD, Jimenez JC, Wilson SE, et al. Complications after laparoscopic gastric bypass: A review of 3464 cases. Arch Surg 2003;138:957-961.

6. Buchwald H, Estok R, Fahrbach K, et al. Trends in mortality in bariatric surgery: A systematic review and meta-analysis. Surgery 2007;142:621-632.

7. Giulianotti PC, Coratti A, Angelini M, et al. Robotics in general surgery: Personal experience in a large community hospital. Arch Surg 2003;138:777-784.

8. Tomulescu V, Stanciulea O, Balescu I, et al. First year experience of robotic-assisted laparoscopic surgery with 153 cases in a general surgery department: Indications, technique and results. Chirurgia (Bucur) 2009;104:141-150.

9. Parini U, Fabozzi M, Brachet Contul R, et al. Laparoscopic gastric bypass performed with the Da Vinci Intuitive Robotic
System: Preliminary experience. Surg Endosc 2006;20:18511857.

10. Fullum TM, Aluka KJ, Turner PL. Decreasing anastomotic and staple line leaks after laparoscopic Roux-en-Y gastric bypass. Surg Endosc 2009;23:1403-1408.

11. Himpens JM. The gastrojejunostomy in laparoscopic Rouxen-Y gastric bypass. Semin Laparosc Surg 2004;11:171-177.

12. Artuso D, Wayne M, Grossi R. Use of robotics during laparoscopic gastric bypass for morbid obesity. JSLS 2005;9: 266-268.

13. Podnos YD, Jimenez JC, Wilson SE, et al. Complications after laparoscopic gastric bypass: A review of 3464 cases. Arch Surg 2003;138:957-961.

14. Bell BJ, Bour ES, Scott JD, et al. Management of complications after laparoscopic Roux-en- $Y$ gastric bypass. Minerva Chir 2009;64:265-276.

15. Flum DR, Salem L, Elrod JA, et al. Early mortality among medicare beneficiaries undergoing bariatric surgical procedures. JAMA 2005;294:1903-1908.

16. Higa KD, Ho T, Boone KB. Laparoscopic Roux-en-Y gastric bypass: Technique and 3-year follow up. J Laparoendosc Adv Surg Tech A 2001;11:377-382.

17. Mohr CJ, Nadzam GS, Curet MJ. Totally robotic Roux-en-Y gastric bypass. Arch Surg 2005;140:779-786.

18. Schauer P, Ikramuddin S, Hamad G, Gourash W. The learning curve for laparoscopic Roux-en- $Y$ gastric bypass is 100 cases. Surg Endosc 2003;17:212-215.

19. Oliak D, Ballantyne GH, Weber P, et al. Laparoscopic Rouxen-Y gastric bypass: Defining the learning curve. Surg Endosc 2003;17:405-408.

20. Yu SC, Clapp BL, Lee MJ, et al. Robotic assistance provides excellent outcomes during the learning curve for laparoscopic Roux-en-Y gastric bypass: Results from 100 roboticassisted gastric bypasses. Am J Surg 2006;192:746-749.

21. Sanchez BR, Mohr CJ, Morton JM, et al. Comparison of totally robotic laparoscopic Roux-en-Y gastric bypass and traditional laparoscopic Roux-en-Y gastric bypass. Surg Obes Relat Dis 2005;1:549-554.

22. Hubens G, Balliu L, Ruppert M, et al. Roux-en-Y gastric bypass procedure performed with the da Vinci robot system: Is it worth it? Surg Endosc 2008;22:1690-1696.

23. Ali MR, Bhaskerrao B, Wolfe BM. Robot-assisted laparoscopic Roux-en-Y gastric bypass. Surg Endosc 2005;19:468-472.

24. Amodeo A, Linares Quevedo A, Joseph JV, et al. Robotic laparoscopic surgery: Cost and training. Minerva Urol Nefrol 2009;61:121-128.

Address correspondence to: Subhashini M. Ayloo, MD, FACS

Division of General, Minimally Invasive and Robotic Surgery Department of Surgery University of Illinois at Chicago 840 South Wood St. (MC 958)

Chicago, IL 60612

E-mail: ayloosub@uic.edu 